Revista Bioética

\title{
PESQUISA
}

\section{Espiritualidade nos cuidados paliativos: questão de saúde pública?}

Mary Esperandio ${ }^{1}$, Carlo Leget ${ }^{2}$

1. Programa de Pós-Graduação em Bioética, Escola de Ciências da Vida, Pontifícia Universidade Católica do Paraná, Curitiba/PR, Brasil. 2. Departamento de Ética em Cuidados, Universidade de Estudos Humanísticos, Utrecht, Holanda.

\section{Resumo}

Reconhecido pela Organização Mundial da Saúde como essencial às boas práticas em assistência paliativa, "cuidado espiritual" é termo recente na saúde brasileira e carece de reflexão específica. A fim de diminuir tal lacuna, este estudo apresenta o estado da arte sobre a temática, traz breves orientações sobre como identificar necessidades espirituais e descreve quatro ferramentas úteis para esse cuidado. Após contextualização, reflete-se, de forma introdutória e com base nos campos da bioética e da teologia pública, se esse tipo de cuidado seria questão de saúde pública. $O$ texto conclui com recomendações para subsidiar políticas públicas voltadas à implementação do cuidado espiritual na assistência paliativa no Brasil.

Palavras-chave: Cuidados paliativos. Espiritualidade. Bioética. Saúde pública. Teologia.

\section{Resumen}

Espiritualidad en los cuidados paliativos: ¿un problema de salud pública?

Aunque reconocido por la Organización Mundial de la Salud como esencial para las buenas prácticas en cuidados paliativos, "cuidado espiritual" es un término reciente en el contexto de la salud brasileña, acerca del cual todavía falta reflexión específica. Para contribuir a llenar este vacío, este estudio presenta una breve guía sobre como identificar las necesidades espirituales, describiendo cuatro herramientas útiles para este cuidado. Después de la contextualización se refleja, de forma introductoria y con base en los campos de la bioética y de la teología pública, si este tipo de cuidado es un tema de salud pública. El texto concluye con recomendaciones que pueden apoyar las políticas públicas orientadas a implementar el cuidado espiritual en la asistencia paliativa en Brasil.

Palabras clave: Cuidados paliativos. Espiritualidad. Bioética. Salud pública. Teología.

\section{Abstract}

Spirituality in palliative care: a public health issue?

Recognized by the World Health Organization as essential for good practices in palliative treatments, "spiritual care" is a recent term in the context of Brazilian healthcare, and should be more well-defined. To fill this gap, this study presents an overview of the updating of spiritual care in Brazil, the United States and Europe, provides brief guidance on how to identify spiritual needs, and outlines four useful tools for this type of care. After the contextualization follows an interdisciplinary and introductory reflection based on bioethics and public theology, questioning whether this care would be a public health issue. The text concludes by presenting some recommendations that may support public policies aimed at implementing spiritual support in palliative care in Brazil.

Keywords: Palliative care. Spirituality. Bioethics. Public health. Theology.

Declaram não haver conflito de interesse. 
A disseminação da filosofia dos cuidados paliativos (CP) e a ampliação de sua oferta (que deve alcançar também a atenção primária nos próximos anos) ${ }^{1}$ permitem debater se o cuidado espiritual (CE) seria questão de saúde pública, uma vez que é essencial na provisão de boas práticas em CP. A problematização é pertinente, haja vista que a dimensão espiritual é reconhecida pela Organização Mundial da Saúde (OMS) como componente intrínseco da assistência paliativa ${ }^{2}$.

No Brasil, as pesquisas sobre espiritualidade nos $\mathrm{CP}$ são ainda muito recentes, mas estão se propagando. $\mathrm{O}$ que estes estudos têm demonstrado? As pesquisas empíricas brasileiras distinguem-se dos trabalhos norte-americanos e europeus, que investigam o tema há mais tempo? Seus resultados subsidiam políticas públicas para implementar o cuidado espiritual nos CP? O CE pode ser considerado questão de saúde pública? E de que modo a bioética e outros campos do saber podem se unir em diálogo interdisciplinar para melhorar a compreensão do tema e desenvolver o conhecimento? Essas perguntas estão na base deste estudo.

\section{Atual estado da arte}

\section{$O$ contexto brasileiro}

Pesquisou-se a produção científica brasileira de abordagem empírica sobre espiritualidade no contexto dos CP no Portal de Periódicos Capes, na Biblioteca Virtual em Saúde, na Scientific Electronic Library Online (SciELO) e no PubMed, utilizando os termos "cuidado espiritual and cuidados paliativos", "espiritualidade and cuidados paliativos" e "bioética". Foram encontrados 18 estudos empíricos publicados entre 2003 e 2018: quatro dissertações, duas teses e 12 artigos. Três dos artigos foram publicados em $2003^{3}, 2006^{4}$ e $2014^{5}$, e os outros nove ${ }^{6-14}$ foram publicados a partir de 2015.

A investigação sobre espiritualidade nos CP é recente, e vem crescendo paralelamente à progressão desse tipo de assistência no país. A maioria dos estudos parece se preocupar apenas em comprovar a relação entre espiritualidade e saúde. É digno de nota que, embora o termo "cuidado espiritual" tenha aparecido com frequência nos artigos publicados a partir de 2015 , somente duas teses ${ }^{15,16}$ e uma dissertação ${ }^{17}$ o definiram. Antes disso, o conceito aparece em texto de 2007 não computado no levantamento, publicado em revista fora de circulação desde $2014^{18}$.

A literatura pesquisada mostra que, no contexto dos $\mathrm{CP}$, a espiritualidade é fonte de sentido para a experiência da doença ${ }^{19}$, produz sensação de bem-estar ${ }^{20}$ e qualidade de vida ${ }^{6,12}$, é recurso de coping (enfrentamento) ${ }^{21}$, suporte para enfermos e familiares ${ }^{22}$ e meio de desenvolvimento e ressignificação da vida ${ }^{8}$. Contudo, profissionais da saúde têm dificuldade em identificar necessidades espirituais e atendê-las ${ }^{12,23}$, e um estudo ${ }^{14}$ identificou que menos de $15 \%$ dos pacientes internados tiveram tais demandas atendidas ou receberam apoio psicológico.

Em geral, a equipe não avalia sofrimento espiritual, uma vez que essa dimensão é confundida com religiosidade, sendo vista como de natureza privada ${ }^{23}$. Poucos profissionais realizam o CE, por meio de práticas como oração, oferta de conforto e ajuda no processo de busca de sentido, tanto para pacientes quanto para a própria equipe multiprofissional ${ }^{12}$. Estudos destacam a carência na formação profissional para integrar esse tipo de assistência nos $\mathrm{CP}^{10,24,25}$.

Entre os trabalhos com foco em prática de CE nos CP, três autores se destacam: Ana Catarina de Araújo Elias ${ }^{3}$, Michell Ângelo Marques Araújo ${ }^{15}$ e Luciana Winterkorn Dezorzi ${ }^{16}$. Elias ${ }^{3}$ criou e testou uma técnica de intervenção denominada Relaxamento, Imagens Mentais e Espiritualidade (Rime), cuja finalidade é ressignificar a dor espiritual de pacientes (crianças, adolescentes e adultos) em CP e familiares. Seis profissionais da área da saúde foram treinados e aplicaram a técnica em 11 pacientes. Os resultados reportados foram positivos tanto para pacientes quanto para profissionais, como promoção de bem-estar, melhora na qualidade de vida durante o processo de morte e ressignificação da dor espiritual ${ }^{4}$.

No estudo-intervenção desenvolvido para sua tese de doutorado, Araújo ${ }^{15}$ dirigiu CE a três pacientes adultos e, com base nisso, construiu um modelo de cuidado sustentado sobre 17 pressupostos teóricos, relacionados a cinco conceitos básicos: ser humano, processo saúde-doença, enfermagem, ambiente e cuidado espiritual.

Já a enfermeira Luciana Dezorzi ${ }^{16}$ desenvolveu em sua tese de doutorado uma proposta de formação para a prática do CE em CP. A autora validou uma escala de competência e a aplicou a 52 profissionais da saúde, antes e depois de participarem de módulo de educação de quatro horas sobre $\mathrm{CE}$ especialmente desenvolvido para trabalhadores envolvidos em CP (mas não restrito a esse grupo) e publicado em forma de cartilha eletrônica ${ }^{26}$. O treinamento abordou noções de espiritualidade, religiosidade, crise e cuidado espiritual, formas de avaliar recursos e necessidades de pacientes e familiares. A autora aponta resultados positivos da intervenção 
educativa, indicando que ajuda a minimizar lacunas da formação ${ }^{16}$. No entanto, a efetividade dessa integração deve ser verificada em futuras pesquisas.

Além desses estudos empíricos, foram encontradas três publicações teóricas ${ }^{27-29}$ relacionadas ao tema. Hefti e Esperandio ${ }^{28}$ apresentam modelo interdisciplinar no contexto da saúde (não exclusivamente nos (P). Para os autores, o CE interdisciplinar caracteriza-se pela avaliação da espiritualidade da pessoa enferma, que pode ser realizada por qualquer componente da equipe multidisciplinar visando integrar essa dimensão no cuidado. O especialista na assistência espiritual (capelão/capelã) faz parte da equipe e contribui com sua especialidade para a provisão das necessidades espirituais tanto de pacientes e familiares como dos profissionais.

No campo da bioética, Salles ${ }^{27}$ não aborda diretamente o CE, mas defende, a partir dos princípios da autonomia e da beneficência, a importância de considerar as crenças religiosas de pacientes em fim de vida. $\mathrm{O}$ autor apresenta crenças sobre a morte presentes no budismo, no espiritismo, em religiões afro-brasileiras (candomblé, umbanda e quimbanda) e no catolicismo. Já Garanito e Cury ${ }^{29}$ discutem o tema especificamente na pediatria, destacando que também a criança apresenta necessidades espirituais. As autoras pontuam a importância do capelão hospitalar e a necessidade de incluir a questão da espiritualidade e da assistência paliativa na formação de profissionais da saúde.

Diferentemente do contexto norte-americano e europeu, no Brasil a assistência espiritual especializada (capelania) é escassa ${ }^{28-30}$. Este cuidado é realizado como "assistência religiosa", quase sempre por agentes voluntários ou líderes religiosos chamados a prestá-la. No levantamento da produção científica sobre CE (ou capelania) no contexto específico dos $\mathrm{CP}$, nenhum estudo foi capturado com os descritores utilizados.

\section{O contexto norte-americano}

Pesquisadores dos Estados Unidos apontam que os enfermos anseiam por CE em fim de vida ${ }^{31,32}$. Também há evidências de que atender necessidades espirituais de pacientes terminais se associa a melhor utilização de hospice, índices mais baixos de mortalidade em unidades de tratamento intensivo, diminuição de tratamentos agressivos (especialmente entre quem usa estratégias de coping), maior qualidade de vida e menor custo hospitalar e risco de depressão ${ }^{33-35}$

Revisão de literatura realizada por dez pesquisadores norte-americanos e publicada em duas partes $^{36,37}$ abordou, na primeira parte ${ }^{36}$, definições de espiritualidade, métodos de pesquisa e instrumentos de avaliação e evidências dos resultados do CE na saúde. Na segunda parte ${ }^{37}$, os pesquisadores levantaram o modo como o tema tem sido tratado, como são realizadas as intervenções e como tem sido a formação de profissionais para esse tipo de abordagem.

Especificamente em relação ao $\mathrm{CE}$ nos $\mathrm{CP}$, os autores sublinham que desde 2009 , na primeira conferência norte-americana de consenso sobre espiritualidade na assistência paliativa, estabeleceram-se parâmetros para a formação da equipe. Os profissionais deveriam: receber formação condizente com sua especialidade; saber realizar anamnese espiritual; conhecer claramente os recursos disponíveis para a pessoa enferma em termos de CE; ser adequadamente treinados para atender as necessidades espirituais do paciente; conhecer o impacto dos valores e crenças espirituais/religiosas sobre as decisões em saúde; conhecer diferentes especialistas em cuidado espiritual e saber quando indicá-los a pacientes e familiares; desenvolver presença compassiva e escuta ativa; e ter formação sobre autorreflexão espiritual e autocuidado ${ }^{36,37}$.

Sobre as intervenções relacionadas às necessidades espirituais, tem-se enfatizado modelos com foco na qualidade de vida, incluindo a psicoterapia, a "revisão de vida" e intervenções multidisciplinares, corporais e mentais ${ }^{37}$. No que diz respeito ao CE provido por assistentes especializados (capelães e capelãs), os pesquisadores observam que é necessária pesquisa quantitativa que indique resultados com mais clareza, haja vista que o trabalho realizado por tais profissionais em geral focaliza o processo, mais do que os resultados ${ }^{36,37}$.

\section{O contexto europeu}

Em levantamento recente da literatura sobre CE (não sobre espiritualidade), Gijsberts e colaboradores ${ }^{38}$ encontraram 53 estudos, somando amostra total de mais de 30 mil participantes. A maioria das pesquisas teve como objeto principal a perspectiva de profissionais de saúde e pacientes sobre CE e a interação entre provedores e receptores desse tipo de cuidado. Muitos estudos têm sido conduzidos no Reino Unido e na Holanda.

Os autores observam que a atenção à espiritualidade na assistência paliativa envolve também a questão da esperança. Cuidadores espirituais (spiritual caregivers) buscam ser uma presença plena, provendo esperança, conforto e paz. Sua prática de cuidado inclui criação, narrativa e trabalho com 
rituais. Para os pesquisadores, profissionais da saúde deveriam olhar para esse assunto não apenas sob perspectiva "realista", mas reconhecer também a perspectiva "funcional" (ligada ao bem-estar) e "narrativa" (história de vida) ${ }^{38}$.

Os resultados dos estudos analisados sugerem que o CE tem efeito positivo sobre a pessoa enferma, aliviando, por exemplo, o desconforto. Contudo, a evidência ainda é baixa. Para implementação efetiva, é preciso desenvolver competência espiritual por meio de educação e autorreflexão, dando mais visibilidade às dimensões espirituais no cuidado em saúde em geral. Nesse contexto, o serviço de capelania desempenha papel importante ${ }^{38}$. Tal como nos estudos norte-americanos ${ }^{37}$, aponta-se a necessidade de pesquisas sobre assistência espiritual especializada.

Enquanto nos Estados Unidos as intervenções em CE estão relacionadas à qualidade de vida e faltam trabalhos baseados em hipóteses a priori, na Europa sente-se a necessidade de, primeiramente, desenvolver pesquisas com resultados mais adequados ${ }^{38}$. Os autores observam que a questão da qualidade de vida pode não capturar totalmente o efeito dos trabalhos criativos, de narrativa e de rituais apontados como formas de prover cuidado espiritual ${ }^{38}$. Enfim, pesquisadoras e pesquisadores europeus e norte-americanos pontuam que é premente também pesquisar o impacto da educação em CE para a saúde da pessoa enferma, da família e da equipe multiprofissional ${ }^{37,38}$.

\section{Espiritualidade e cuidado espiritual}

Definir cuidado espiritual e espiritualidade não é tarefa simples, uma vez que esses conceitos estão sujeitos a contínuo debate ${ }^{36,37,39}$. Leget $^{39}$ destaca que a dificuldade de consenso se relaciona tanto com as diferenças culturais e acadêmicas quanto com o método usado para delimitar os termos. No entanto, esse é ponto de partida necessário, mesmo sabendo que as definições serão temporárias.

\section{Definindo os termos}

Dos 18 estudos brasileiros publicados entre 2003 e 2018, 14 (77,8\%) definiram "espiritualidade" como medida de produção de sentido e propósito existencial, algo mais amplo do que "religiosidade". Boa parte dos estudos publicados no Brasil segue a definição de consenso global, estabelecida em duas conferências internacionais ocorridas em 2012 e 2013: espiritualidade é aspecto dinâmico e intrínseco da humanidade por meio do qual as pessoas buscam sentido último, propósito e transcendência, experienciam a relação consigo mesmas, com a família, com outros, com a comunidade, a sociedade, a natureza e o significante ou sagrado. A espiritualidade é manifestada por crenças, valores, tradições e práticas ${ }^{40}$.

Nas teses e dissertações que definiram "cuidado espiritual", o termo foi considerado sinônimo de cuidado transpessoal ${ }^{17}$. Essencialmente relacional, o CE teria como foco central a busca e a descoberta do sentido da vida ${ }^{41}$ para realização plena e para o fim do desespero, reconhecendo e respondendo às necessidades do espírito humano, especialmente quando confrontado com traumas, problemas de saúde ou tristeza. (...) [Ele] começa com o contato humano encorajador e com um relacionamento compassivo (...), capaz de se mover na direção que a necessidade requer ${ }^{42}$.

Cuidado espiritual não é sinônimo de "cuidado religioso". Podemos defini-lo como tipo de assistência fundamentado na identificação e atenção a demandas ligadas à espiritualidade. Ou seja, o CE inclui ajudar as pessoas na busca por sentido, propósito, esperança e conexão em situações que parecem profundamente sem sentido ou esperança, como em caso de doença grave, progressiva e que ameaça a continuidade da existência. Cuidado espiritual implica olhar acurado, escuta atenta e compassiva, que acolhe a pessoa que sofre, buscando minimizar seu sofrimento, seja ele físico, emocional, psicossocial ou espiritual.

A crítica segundo a qual "espiritualidade" é conceito vago parece comprometer a prática do CE. Contudo, como apontam Swinton e Pattison ${ }^{43}$, é justamente na imprecisão do termo que reside sua força, pois fala daquilo que, em geral, está ausente ou é pouco reconhecido nos cuidados em saúde. Concordando com esses autores, salienta-se aqui que a espiritualidade expressa algo do processo existencial que é condicionado ao tempo histórico, ao espaço geográfico, à cultura e à linguagem, além das condições sociais e econômicas. Há algo que sempre escapa à conceitualização, localizando e datando as definições e, consequentemente, as práticas de cuidado espiritual, expressando em sua forma as condições de sua emergência.

Além destes condicionantes, a identificação das necessidades espirituais depende da atitude e da competência teórica e técnica do profissional de saúde. Essas necessidades podem ser identificadas de muitas maneiras, em muitos níveis, e são inseparáveis das dimensões físicas, psicossociais e existenciais, como pontua Leget ${ }^{39}$. Isto, porém, não significa que a pessoa sempre as tem. 


\section{Como identificar necessidades espirituais}

Leget $^{39}$ observa que as necessidades espirituais são explícitas, implícitas ou até mesmo ocultas, podendo inclusive se interligar a outros tipos de necessidade. Kellehear ${ }^{44}$ mostrou, com base em revisão de literatura, que a espiritualidade se evidencia em necessidades multidimensionais, descritas em três categorias: situacionais (ligadas à busca de propósito e sentido, esperança, significado e afirmação, mutualidade, conexão, representação social); morais e biográficas (de paz e reconciliação, conexão com os outros, oração, análise moral e social, perdão, solução de pendências e encerramento da biografia); e religiosas (reconciliação religiosa, perdão e apoio divino, direitos religiosos/sacramentos, visitas do clero, discussões sobre Deus, vida eterna e esperança) ${ }^{44}$.

Leget $^{39}$ complementa que as necessidades espirituais são expressas de variadas maneiras, de acordo com o caráter e a cultura de cada pessoa. Para algumas, a espiritualidade está ligada ou sobreposta à religiosidade, enquanto para outras ela é vivida de maneira intelectual, por meio do estudo e da leitura de livros, da discussão e da busca por entendimento em quadro de referência mais amplo. Há aquelas que compreendem a busca espiritual como uma jornada para a interioridade, usando meditação ou outros modos mais voltados à experiência interior. Outro grupo pode estar mais focado em ações e organizações, ajudando outras pessoas ou contribuindo para melhorar o mundo. Identificar necessidades espirituais é crucial para prover o CE. Neste sentido, é preciso conhecer ferramentas e desenvolver competências que ajudem a reconhecê-las e atendê-las.

\section{Ferramentas para avaliar a espiritualidade nos cuidados paliativos}

Quais são as melhores ferramentas disponíveis para avaliar a espiritualidade/religiosidade de modo a facilitar o CE nos CP? Em revisão sistemática, Lucchetti, Bassi e Lucchetti ${ }^{45}$ identificaram 25 instrumentos para uso clínico ou de pesquisa, apontando cinco deles como os mais apropriados: FICA, SPIRITual History, FAITH, HOPE e Royal College of Psychiatrists.

Blaber, Jone e Willis ${ }^{46}$ analisaram FICA, SPIRITual, FAITH e HOPE, considerando seus pontos fortes e fracos a partir de cinco critérios: 1) foco na pessoa enferma; 2) concisão; 3) flexibilidade; 4) facilidade de memorização; e 5) confiabilidade (abordagem que não seja do tipo "checagem de itens"). Os autores apontaram HOPE como o instrumento mais apropriado para uso nos $\mathrm{CP}$, porque ajuda o profissional a traçar planos individualizados para o CE contínuo de cada paciente. Contudo, FICA foi o único a ser validado.

A seguir são apresentados os quatro instrumentos de abordagem prática considerados os mais adequados para o contexto aqui estudado. Desenvolvido no contexto da medicina de família ${ }^{47}$, o instrumento HOPE avalia a dimensão da espiritualidade com base em quatro componentes, indicados em seu nome. O primeiro componente (a letra " $h$ ", de "hope", "esperança") busca responder quais são as fontes de esperança, força, conforto e paz do paciente, no que ele se agarra em tempos dificeis e o que o sustenta e lhe possibilita continuar lutando, apesar das dificuldades. O segundo elemento (letra "o") procura identificar se o paciente se considera parte de uma religião organizada, e até que ponto ela é importante e útil para o enfermo.

Já o terceiro elemento (letra " $p$ ") questiona práticas pessoais - se o respondente tem crenças independentemente de religião organizada, se acredita em Deus e que aspectos de sua prática espiritual (meditação, oração, leitura de textos religiosos, músicas, frequência em serviços religiosos etc.) considera mais úteis. Por fim, o último elemento (letra " $\mathrm{e}$ ") trata do efeito das questões relativas ao final de vida sobre os cuidados médicos - por exemplo, se o paciente se preocupa com algum conflito entre suas crenças espirituais/religiosas e decisões relativas ao tratamento de saúde ${ }^{47}$.

O segundo instrumento, o acrônimo FICA, proposto por Christina Puchalski ${ }^{48}$, visa identificar valores e crenças, mecanismos de enfrentamento e sistemas de apoio que aparelhem a oferta de cuidado compassivo. São feitas diversas perguntas para levantar aspectos sobre a fé e sua importância para o paciente, a fim de fornecer suporte religioso e atender necessidades espirituais. As perguntas referentes a cada letra são:

- $F$ (fé): você tem crenças espirituais/religiosas que ajudam a lidar com situações de sofrimento? Caso a pessoa não se identifique com algum sistema de crenças, o profissional pode perguntar: o que dá sentido à sua vida?

- I (importância): qual é a importância da espiritualidade no enfrentamento da enfermidade e qual o papel de suas crenças sobre decisões em saúde?

- $\quad C$ (comunidade religiosa): qual é a sua igreja ou comunidade de fé? Ela te dá suporte? De que modo? Existe algum grupo que você realmente ame e que funcione como apoio para você?

- $A$ (atendimento): como podemos atender suas necessidades espirituais? Há algo que você gostaria que fosse feito para atendê-las? 
A terceira ferramenta apresentada, disponibilizada pelo Mount Vernon Cancer Network em 2007, tem sido bastante utilizada por profissionais da saúde na Europa ${ }^{39}$. O instrumento se baseia em três perguntas cuja finalidade é verificar aspectos básicos relacionados ao sentido, aos recursos e ao suporte espiritual: 1) como você entende o que está acontecendo e que sentido essa experiência tem para você?; 2) quais recursos você emprega quando a vida está difícil, e o que te ajuda em tempos dificeis?; 3) você julga que seria útil conversar com alguém sobre questões espirituais e de fé?

Por fim, o último instrumento é composto por uma única pergunta: o que eu preciso saber sobre você como pessoa para cuidar de você da melhor maneira possível ${ }^{49}$ ? A questão expressa preocupação centrada no indivíduo, deixando-Ihe a decisão de informar ao cuidador o que considera apropriado.

\section{Bioética, cuidado espiritual e saúde pública}

As evidências empíricas do impacto da espiritualidade/religiosidade deveriam ser suficientes para mudar o cuidado em saúde em pelo menos duas instâncias. A primeira diz respeito ao discurso de profissionais sobre a carência na formação ou falta de tempo para integrar o CE em sua prática ${ }^{50,51}$. A segunda se refere às políticas públicas.

Quanto à carência na formação profissional, é fato que no Brasil poucos cursos oferecem conteúdos sobre espiritualidade ${ }^{52}$. Todavia, o volume significativo de estudos sobre o tema precisa ser mais bem utilizado pelos profissionais. A falta de tempo ${ }^{50,51,53}$ remete a questões estruturais do sistema público de saúde e ao modo como este se organiza, revelando o "preço da vida". Na sociedade capitalista, a "mercadoria" tempo é dimensão importante de políticas, estruturas e sistemas econômicos ${ }^{43}$. Assim, a justificativa não é politicamente neutra, uma vez que o cuidado espiritual é condicionado pelo tempo demandado. Remete-se a um "cálculo" da vida e de prioridades - dedica-se tempo ao que mais se valoriza. Esse afastamento da dimensão humana das práticas de cuidado denuncia algo de incongruente nas políticas em saúde.

A dimensão da espiritualidade não pode ser ignorada, pois ela permite construir sentido para o sofrimento de enfrentar uma doença grave e que ameaça a continuidade da existência. A prática do $C E$ promove a dignidade da pessoa, melhora a qualidade de vida, aumenta a sensação de bem-estar e dá maior clareza quanto a decisões importantes nesse cenário.
Por seu caráter essencialmente interdisciplinar e seus referentes éticos, a bioética é o campo aglutinador da discussão sobre cuidado espiritual como questão de saúde pública. Há preocupações e enfoques em comum entre o campo da bioética (global ${ }^{54}$, narrativa $^{9,55}$ e de proteção ${ }^{56}$ ) e a teologia pública ${ }^{57,58}$.

Leo Pessini ${ }^{54}$ destaca solidariedade, vulnerabilidade e precaução ao discorrer sobre a contribuição da bioética global. A bioética de proteção destaca tanto as condições de possibilidade de uma bioética sanitária ${ }^{59}$ quanto o diálogo interdisciplinar sobre a qualidade de vida das populações humanas. "Proteção", nesse sentido, não significa a relação paternalista entre quem presta o cuidado e quem o recebe passivamente. $O$ termo se refere ao cuidado de populações de vulnerados, considerando a vulnerabilidade condição própria do existir ${ }^{56}$. A "vulneração" instala-se quando não há condições plenas para exercer a autonomia. Nos CP, a autonomia é prejudicada em razão de fatores que podem exacerbar o sofrimento e impactar a tomada de decisões.

A teologia pública também enriquece 0 debate, pois evidencia a solidariedade ${ }^{57,58}$, a compaixão e a ética do cuidado ${ }^{58}$, apontando o papel fundamental de quem presta o CE - em geral, pessoas formadas em teologia ${ }^{49,58}$. Por vezes, pacientes e familiares se sentem mais à vontade para discutir decisões éticas com quem provê esse tipo de assistência do que com profissionais de saúde, comprovando a importância dessa especialidade na equipe multidisciplinar - importância que tende a não ser reconhecida no Brasil.

Capelães poderiam contribuir singular e significativamente com o enfermo, sua família e a própria equipe. Pode-se ilustrar esse apoio com narrativa registrada em estudo que investigou princípios e valores implicados na prática da sedação paliativa (sublinha-se que o foco principal não era a espiritualidade, a qual emergiu nos resultados) ${ }^{60}$. A pesquisa avalia o caso de um paciente com dor extrema que decide não se submeter a sedação paliativa, provavelmente por convicções religiosas. A pessoa que narra o caso afirma se sentir frustrada com a decisão, mas a aceita em nome de princípios bioéticos como respeito à autonomia e a valores religiosos: se o doente (...) quer morrer com sofrimento, trabalhamos a equipe e se respeita a decisão dele ${ }^{61}$. Outra entrevistada diz: e aí acaba que quem sofre é quem tem todos os princípios dos CP e que acreditaria que se fizesse alguma coisa poderia aliviar o sofrimento ${ }^{61}$.

Vários questionamentos poderiam ser levantados, mas importa destacar, a partir dos referentes da bioética de proteção, as seguintes questões: 1) não 
estaria o paciente vulnerado, dado seu sofrimento extremo, reconhecido pelo próprio profissional?; 2) nessa situação, é possível exercer plenamente a autonomia?; 3) morrer com sofrimento seria de fato opção ou expressaria conflito espiritual oculto, e portanto uma necessidade a ser atendida?; 4) o sofrimento expresso pelo profissional também deveria ser objeto de medicação?; 5) a equipe tem mesmo "todos os princípios dos CP" para aliviar o sofrimento?; e 6) o CE, tanto da pessoa enferma quanto da equipe, foi incluído na assistência paliativa? O exemplo mostra a relevância da espiritualidade nos $\mathrm{CP}$, reforçando a necessidade de debater o tema como questão de saúde pública ${ }^{56}$.

Por sua vez, a bioética narrativa ${ }^{9,60}$ tem como fundamento a "história de vida" contada pela pessoa enferma, a fim de buscar elementos que justifiquem e deem sentido à tomada de decisões. As questões éticas no contexto dos CP estão intimamente ligadas a crenças e valores que norteiam escolhas e definem comportamentos ${ }^{55}$. Por sua característica de escuta ativa e valorização do aspecto relacional, a bioética narrativa promove a dignidade humana, valorizando o passado e o presente, considerando o modo como a história pessoal expressa sentido e propósito e possibilitando uma reflexão mais profunda nas deliberações sobre o tratamento.

No contexto dos $\mathrm{CP}$, a pessoa enferma enfrenta não apenas a dor física advinda dos sintomas de doença grave. Ela experiencia um sofrimento multifatorial que extrapola os limites do corpo físico e se manifesta em problemas relacionais, crises e conflitos espirituais não tratáveis com medicamentos e que podem agravar a percepção da dor. Cicely Saunders ${ }^{62}$ caracterizou essa experiência como "dor total", pois engloba, para além do componente físico, o sofrimento psicológico, social, emocional e espiritual.

A busca de sentido para o sofrimento se situa no contexto da história de vida da pessoa. Do mesmo modo, decisões éticas não são isoladas do contexto relacional e social da unidade de tratamento (paciente e familiares), como apontam Muldoon e King ${ }^{55}$. Nas narrativas do enfermo estão presentes seus valores e crenças, aquilo que o ajuda a encontrar sentido e embasa suas decisões.

A bioética narrativa, a teologia pública e o cuidado espiritual têm em comum o senso de responsabilidade e a compaixão pela pessoa que sofre, a promoção da dignidade por meio do relacionamento e técnicas que valorizam a história de vida e ajudam a construir sentido e se conectar consigo mesma, com outros e com aquilo que a pessoa considera sagrado.

\section{Das barreiras à potencialidade}

A organização de serviços de CP no Brasil deve levar em conta a realidade sociocultural do país, onde a religiosidade, enquanto expressão da espiritualidade, é importante para a subjetividade da população. Os dados do último censo do Instituto Brasileiro de Geografia e Estatística ${ }^{63}$ mostram que, embora o mapa religioso brasileiro tenha se modificado, a população continua altamente religiosa. No Brasil, $64,6 \%$ da população é católica; $22,2 \%$ são pentecostais; $2 \%$ espíritas; $0,3 \%$ professam religiões afro-brasileiras; e 2,7\% praticam outras crenças. Pela primeira vez aparece um grupo que se autodeclara "sem religião" (8\%), e emerge outro grupo (5\%) que se afirma com "dupla" ou "múltipla pertença" ${ }^{63}$. O crescente número de pessoas sem religião indica a necessidade de considerar a espiritualidade para além dela.

Crenças e valores (de pacientes, familiares e profissionais) influenciam os resultados em saúde. Estudos brasileiros ${ }^{50,51,53}$ apontam que pacientes expressam o desejo de que a espiritualidade seja integrada no tratamento em saúde, e profissionais reconhecem seu impacto no tratamento. Entretanto, as políticas de saúde pública do país parecem ignorar esses dados. Para reverter esse quadro, tendo por base a literatura consultada, é possível fazer algumas recomendações para integrar o CE focando quatro eixos: 1) teoria e pesquisa; 2) práticas de cuidado; 3) educação; e 4) política organizacional.

No eixo da teoria e pesquisa científica, é preciso construir e difundir modelos de CE com fundamentação teórico-prática e culturalmente sensíveis, a fim de que a assistência tenha propósito claro e adequado para responder às reais necessidades de pacientes e familiares. Ademais, deve-se expandir a pesquisa de temas como tomada de decisão, luto, comunicação de notícias difíceis, CE na atenção primária à saúde e em home care, modelos de cuidado, assistência espiritual especializada e sua participação na equipe multidisciplinar, dignidade, esperança, manifestações antecipadas de vontade, formação de profissionais etc. Por fim, é necessário estimular a cooperação internacional entre pesquisadores.

Já no eixo de práticas de cuidado, o foco é fomentar o uso de ferramentas para conhecer a história de vida do paciente e identificar suas necessidades espirituais. Pode-se providenciar espaço no prontuário médico para reportar a avaliação da espiritualidade/religiosidade, o plano de cuidado proposto e a evolução da assistência espiritual. Outro objetivo desse eixo é estimular a implementação gradativa do CE em equipes de CP. 
No eixo da educação é preciso fornecer treinamento específico para profissionais que já atuam na assistência paliativa, incluindo aspectos como identificação de necessidades espirituais/religiosas implícitas e explícitas, modelos de CE e quando e como encaminhar pacientes e familiares para assistência espiritual especializada. Outros objetivos são: integrar o CE nos CP em currículos dos cursos da área da saúde, treinando olhos, ouvidos e coração de futuros profissionais; oferecer cursos específicos sobre o tema para pessoas formadas em teologia; e conscientizar sobre a importância e o papel da assistência espiritual especializada.

No eixo da política organizacional, é necessário chamar atenção para o potencial da teologia em formar especialistas em assistência espiritual em CP. Para isso, é essencial que disciplina específica sobre CE na assistência paliativa integre a grade curricular dos cursos de bacharelado da área. Também é preciso construir relacionamento de colaboração e exercício interdisciplinar entre cuidadores espirituais e profissionais de saúde, bem como integrar profissional competente como "especialista em assistência espiritual" em equipes multidisciplinares de CP. Por fim, pode-se organizar reuniões nacionais para compartiIhar experiências, construir pontes entre pesquisa e prática e planejar estratégias conjuntas para implementar o CE como parte de políticas públicas.

\section{Considerações finais}

O debate sobre CE como questão de saúde pública está apenas começando. Uma vez que a espiritualidade é tida pela OMS como um dos componentes intrínsecos das boas práticas em assistência paliativa e considerando ainda a resolução governamental que trata da implementação dos CP na atenção primária, essa reflexão é imprescindível. Com base nela é possível efetivamente integrar a dimensão espiritual nesse tipo de assistência, melhorando a qualidade do cuidado tanto dos que estão encerrando sua biografia quanto daqueles que os acompanham. Diferentes áreas são convidadas a desenvolver estudos sobre o tema, produzindo evidências que levem à transformação das políticas públicas e da produção teórico-prática de uma bioética do cuidado, de modo que o cuidado integral e centrado na pessoa seja ponto de partida e chegada.

\section{Referências}

1. Brasil. Ministério da Saúde. Resolução no 41, de 31 de outubro de 2018. Dispõe sobre as diretrizes para a organização dos cuidados paliativos, à luz dos cuidados continuados integrados, no âmbito do Sistema Único de Saúde (SUS). Diário Oficial da União [Internet]. Brasília, p. 276, 31 out 2018 [acesso 25 jun 2019]. Disponível: https://bit.ly/2ChxQhc

2. Worldwide Palliative Care Alliance. Global atlas of palliative care at the end of life [Internet]. Geneva: World Health Organization; 2014 [acesso 25 jun 2019]. Disponível: https://bit.ly/30fT6Mi

3. Elias ACA. Re-significação da dor simbólica da morte: relaxamento mental, imagens mentais e espiritualidade. Psicol Cienc Prof [Internet]. 2003 [acesso 22 nov 2019];23(1):92-7. DOI: 10.1590/S1414-98932003000100013

4. Elias ACA, Giglio JS, Pimenta CAM, El-Dash LG. Therapeutical intervention, relaxation, mental images, and spirituality (RIME) for spiritual pain in terminal patients: a training program. Scientific World Journal [Internet]. 2006 [acesso 27 nov 2019];6:2158-69. DOI: 10.1100/tsw.2006.345

5. Paiva CE, Paiva BSR, Yennurajalingam S, Hui D. The impact of religiosity and individual prayer activities on advanced cancer patients' health: is there any difference in function of whether or not receiving palliative anti-neoplastic therapy? J Relig Health [Internet]. 2014 [acesso 27 nov 2019];53(6):1717-27. DOI: 10.1007/s10943-013-9770-6

6. Camargos MG, Paiva CE, Barroso EM, Carneseca EC, Paiva BSR. Understanding the differences between oncology patients and oncology health professionals concerning spirituality/religiosity: a cross-sectional study. Medicine [Internet]. 2015 [acesso 2 jun 2019];94(47):e2145. DOI: 10.1097/ MD.0000000000002145

7. Ferreira AGC, Duarte TMM, Silva AF, Bezerra MR. Concepções de espiritualidade e religiosidade e a prática multiprofissional em cuidados paliativos. Rev Kairós [Internet]. 2015 [acesso 27 nov 2019];18(3):227-44. DOI: 10.23925/2176-901X.2015v18i3p227-244

8. Paiva BSR, Carvalho AL, Lucchetti G, Barroso EM, Paiva CE. "Oh, yeah, I'm getting closer to god": spirituality and religiousness of family caregivers of cancer patients undergoing palliative care. Support Care Cancer [Internet]. 2015 [acesso 27 nov 2019];23(8):2383-9. DOI: 10.1007/s00520-015-2604-1

9. Manchola C, Brazão E, Pulschen A, Santos M. Cuidados paliativos, espiritualidade e bioética narrativa em unidade de saúde especializada. Rev. bioét. (Impr.) [Internet]. 2016 [acesso 27 nov 2019];24(1):165-75. DOI: 10.1590/1983-80422016241118 
10. Evangelista CB, Lopes MEL, Costa SFG, Abrão FMS, Batista PSS, Oliveira RC. Spirituality in patient care under palliative care: a study with nurses. Esc Anna Nery [Internet]. 2016 [acesso 27 nov 2019];20(1):176-82. DOI: 10.5935/1414-8145.20160023

11. Benites AC, Neme CMB, Santos MA. Significados da espiritualidade para pacientes com câncer em cuidados paliativos. Estud Psicol [Internet]. 2017 [acesso 27 nov 2019];34(2):269-79. DOI: 10.1590/1982-02752017000200008

12. Matos TDS, Meneguin S, Ferreira MLS, Miot HA. Qualidade de vida e coping religioso-espiritual em pacientes sob cuidados paliativos oncológicos. Rev Latinoam Enferm [Internet]. 2017 [acesso 2 jun 2019];25:e2910. DOI: 10.1590/1518-8345.1857.2910

13. Miqueletto M, Silva L, Figueira CB, Santos MR, Szylit R, Ichikawa CRF. Espiritualidade de famílias com um ente querido em situação de final de vida. Rev Cuidarte [Internet]. 2017 [acesso 27 nov 2019];8(2):1616-27. DOI: 10.15649/cuidarte.v8i2.391

14. Arrieira ICO, Thofehrn MB, Porto AR, Moura PMM, Martins CL, Jacondino MB. Espiritualidade nos cuidados paliativos: experiência vivida de uma equipe interdisciplinar. Rev Esc Enferm USP [Internet]. 2018 [acesso 25 jun 2019];52:e03312. DOI: 10.1590/s1980-220x2017007403312

15. Araújo MÂM. O cuidado espiritual: um modelo à luz da análise existencial e da relação de ajuda [tese] [Internet]. Fortaleza: Universidade Federal do Ceará; 2011 [acesso 27 nov 2019]. Disponível: https://bit.ly/3fBGRQq

16. Dezorzi LW. Espiritualidade na atenção a pacientes em cuidados paliativos e os processos de educação dos profissionais de saúde [tese] [Internet]. Porto Alegre: Universidade Federal do Rio Grande do Sul; 2016 [acesso 22 nov 2019]. Disponível: https://bit.ly/2WsOv8e

17. Resende TM. Acupuntura para pacientes oncológicos ambulatoriais: um cuidado espiritual de enfermagem [dissertação] [Internet]. Juiz de Fora: Universidade Federal de Juiz de Fora; 2014 [acesso 27 nov 2019]. Disponível: https://bit.ly/32on4QJ

18. Selli L, Alves J. O cuidado espiritual ao paciente terminal no exercício da enfermagem e a participação da bioética. Bioethikos [Internet]. 2007 [acesso 27 nov 2019];1(1):43-52. Disponível: https://bit.ly/391tI0I

19. Tomaszewski AS, Oliveira SG, Arrieira ICO, Cardoso DH, Sartor SF. Manifestações e necessidades referentes ao processo de morte e morrer: perspectiva da pessoa com câncer. Rev Pesqui Cuid Fundam [Internet]. 2017 [acesso 27 nov 2019];9(3):705-16. DOI: 10.9789/2175-5361.2017.v9i3.705-716

20. Rezende VL, Derchain S, Botega NJ, Sarian LO, Vial DL, Morais SS et al. Avaliação psicológica dos cuidadores de mulheres com câncer pelo General Comfort Questionnaire. Paidéia [Internet]. 2010 [acesso 27 nov 2019];20(46):229-37. DOI: 10.1590/\$0103-863X2010000200010

21. Lima CP, Machado MA. Cuidadores principais ante a experiência da morte: seus sentidos e significados. Psicol Ciênc Prof [Internet]. 2018 [acesso 27 nov 2019];38(1):88-101. DOI: 10.1590/1982-3703002642015

22. Medeiros WCM. A clínica psicológica e a experiência da espiritualidade de pacientes em cuidados paliativos [dissertação] [Internet]. Recife: Universidade Católica de Pernambuco; 2012 [acesso 2 jun 2019]. Disponível: https://bit.ly/2DGLoD8

23. Marinho SO. Cuidados paliativos e práticas de saúde: um estudo sobre a gestão da morte na sociedade contemporânea [tese] [Internet]. Rio de Janeiro: Universidade do Estado do Rio de Janeiro; 2010 [acesso 27 nov 2019]. Disponível: https://bit.ly/2DFEyOf

24. Rocha RCNP. Experiências e necessidades espirituais do familiar cuidador de paciente em atenção paliativa oncológica [dissertação] [Internet]. Niterói: Universidade Federal Fluminense; 2017 [acesso 27 nov 2019]. Disponível: https://bit.ly/2B34r9M

25. Zaccara AAL. Cuidados paliativos e espiritualidade: estudo com residentes da área da saúde [dissertação] [Internet]. João Pessoa: Universidade Federal da Paraíba; 2014 [acesso 27 nov 2019]. Disponível: https://bit.ly/2Ou2Exw

26. Dezorzi LW, Raymundo MM, Goldim JR. Espiritualidade na atenção a pacientes/famílias em cuidados paliativos: um guia de apoio para profissionais de saúde [Internet]. Porto Alegre: WWLivros; 2016 [acesso 25 jun 2019]. Disponível: https://bit.ly/32nK825

27. Salles AA. Bioética e processos de religiosidade entre os pacientes com doenças terminais no Brasil. Rev. bioét. (Impr.) [Internet]. 2014 [acesso 27 nov 2019];22(3):397-406. DOI: $10.1590 / 1983-80422014223021$

28. Hefti R, Esperandio MRG. The interdisciplinary spiritual care model: a holistic approach to patient care. Horizonte [Internet]. 2016 [acesso 27 nov 2019];14(41):13-47. DOI: 10.5752/P.2175-5841.2016v14n41p13

29. Garanito MP, Cury MRG. A espiritualidade na prática pediátrica. Rev. bioét. (Impr.) [Internet]. 2016 [acesso 27 nov 2019];24(1):49-53. DOI: 10.1590/1983-80422016241105

30. Gentil RC, Guia BP, Sanna MC. Organização de serviços de capelania hospitalar: um estudo bibliométrico. Esc Anna Nery [Internet]. 2011 [acesso 27 nov 2019];15(1):162-70. DOI: 10.1590/ S1414-81452011000100023

31. Best $\mathrm{M}$, Butow $\mathrm{P}$, Olver I. Do patients want doctors to talk about spirituality? A systematic literature review. Patient Educ Couns [Internet]. 2015 [acesso 27 nov 2019];98(11):1320-8. DOI: 10.1016/j.pec.2015.04.017

32. MacLean CD, Susi B, Phifer N, Schultz L, Bynum D, Franco M et al. Patient preference for physician discussion and practice of spirituality: results from a multicenter patient survey. J Gen Intern Med [Internet]. 2003 [acesso 27 nov 2019];18(1):38-43. DOI: 10.1046/j.1525-1497.2003.20403.x 
33. Balboni TA, Paulk ME, Balboni MJ, Phelps AC, Loggers ET, Wright AA et al. Provision of spiritual care to patients with advanced cancer: associations with medical care and quality of life near death. J Clin Oncol [Internet]. 2010 [acesso 27 nov 2019];28(3):445-52. DOI: 10.1200/JCO.2009.24.8005

34. Balboni T, Balboni M, Paulk ME, Phelps A, Wright A, Peteet J et al. Support of cancer patients' spiritual needs and associations with medical care costs at the end of life. Cancer [Internet]. 2011 [acesso 27 nov 2019];117(23):5383-91. DOI: 10.1002/cncr.26221

35. Pearce MJ, Coan AD, Herndon JE, Koenig HG, Abernethy AP. Unmet spiritual care needs impact emotional and spiritual well-being in advanced cancer patients. Support Care Cancer [Internet]. 2012 [acesso 27 nov 2019];20(10):2269-76. DOI: 10.1007/s00520-011-1335-1

36. Steinhauser KE, Fitchett G, Handzo GF, Johnson KS, Koenig HG, Pargament Kl et al. State of the science of spirituality and palliative care research part I: definitions, measurement, and outcomes. J Pain Symptom Manag [Internet]. 2017 [acesso 27 nov 2019];54(3):428-40. DOI: 10.1016/j.jpainsymman.2017.07.028

37. Balboni TA, Fitchett G, Handzo GF, Johnson KS, Koenig HG, Pargament KI et al. State of the science of spirituality and palliative care research part II: screening, assessment, and interventions. J Pain Symptom Manag [Internet]. 2017 [acesso 27 nov 2019];54(3):441-53. DOI: 10.1016/j.jpainsymman.2017.07.029

38. Gijsberts MJHE, Liefbroer Al, Otten R, Olsman E. Spiritual care in palliative care: a systematic review of the recent European literature. Med Sci [Internet]. 2019 [acesso 27 nov 2019];7(2):25. DOI: $10.3390 /$ medsci7020025

39. Leget C. Spirituality in palliative care. In: MacLeod RD, Van den Block L, editores. Textbook of palliative care [Internet]. Cham: Springer International Publishing; 2018 [acesso 17 jun 2019]. p. 1-11. Disponível: https://bit.ly/2WqnQZQ

40. Puchalski CM, Vitillo R, Hull SK, Reller N. Improving the spiritual dimension of whole person care: reaching national and international consensus. J Palliat Med [Internet]. 2014 [acesso 27 nov 2019];17(6):642-56. p. 646. Tradução livre. DOI: 10.1089/jpm.2014.9427

41. Araújo MÂM. Op. cit. p. 125.

42. Dezorzi LW. Op. cit. p. 31.

43. Swinton J, Pattison S. Moving beyond clarity: towards a thin, vague, and useful understanding of spirituality in nursing care. Nurs Philos [Internet]. 2010 [acesso 27 nov 2019];11(4):226-37. DOI: $10.1111 / \mathrm{j} .1466-769 X .2010 .00450 . x$

44. Kellehear A. Spirituality and palliative care: a model of needs. Palliat Med [Internet]. 2000 [acesso 27 nov 2019];14(2):149-55. DOI: 10.1191/026921600674786394

45. Lucchetti G, Bassi RM, Lucchetti ALG. Taking spiritual history in clinical practice: a systematic review of instruments. Explore [Internet]. 2013 [acesso 27 nov 2019];9(3):159-70. DOI: 10.1016/j.explore.2013.02.004

46. Blaber M, Jone J, Willis D. Spiritual care: which is the best assessment tool for palliative settings? Int J Palliat Nurs [Internet]. 2015 [acesso 27 nov 2019];21(9):430-8. DOI: 10.12968/ijpn.2015.21.9.430

47. Anandarajah G, Hight E. Spirituality and medical practice: using the HOPE questions as a practical tool for spiritual assessment. Am Fam Physician [Internet]. 2001 [acesso 27 nov 2019];63(1):81-9. Disponivel: https://bit.ly/2OtdHav

48. Puchalski C. Spirituality in health: the role of spirituality in critical care. Crit Care Clin [Internet]. 2004 [acesso 27 nov 2019];20(3):487-504. DOI: 10.1016/j.ccc.2004.03.007

49. Johnston B, Gaffney M, Pringle J, Buchanan D. The person behind the patient: a feasibility study using the patient dignity question for patients with palliative care needs in hospital. Int J Palliat Nurs [Internet]. 2015 [acesso 27 nov 2019];21(2):71-7. DOI: 10.12968/ijpn.2015.21.2.71

50. Esperandio MRG, Machado GAS. Brazilian physicians' beliefs and attitudes toward patients' spirituality: implications for clinical practice. J Relig Health [Internet]. 2019 [acesso 27 nov 2019];58(4):1172-87. DOI: 10.1007/s10943-018-0707-y

51. Lucchetti G, Ramakrishnan P, Karimah A, Oliveira GR, Dias A, Rane A et al. Spirituality, religiosity, and health: a comparison of physicians' attitudes in Brazil, India, and Indonesia. Int J Behav Med [Internet]. 2016 [acesso 27 nov 2019];23(1):63-70. DOI: 10.1007/s12529-015-9491-1

52. Lucchetti G, Oliveira LR, Koenig HG, Leite JR, Lucchetti ALG. Medical students, spirituality and religiosity: results from the multicenter study SBRAME. BMC Med Educ [Internet]. 2013 [acesso 27 nov 2019];13(1):162. DOI: 10.1186/1472-6920-13-162

53. Esperandio MRG. Teologia e a pesquisa sobre espiritualidade e saúde: um estudo piloto entre profissionais da saúde e pastoralistas. Horizonte [Internet]. 2014 [acesso 27 nov 2019];12(35):805-32. DOI: $10.5752 / P .2175-5841.2014 v 12 n 35 p 805$

54. Pessini L. Elementos para uma bioética global: solidariedade, vulnerabilidade e precaução. Thaumazein [Internet]. 2017 [acesso 27 nov 2019];10(19):75-85. Disponível: https://bit.ly/2ZwQ6fc

55. Muldoon M, King N. Spirituality, health care, and bioethics. J Relig Health [Internet]. 1995 [acesso 27 nov 2019];34(4):329-50. DOI: 10.1007/bf02248742

56. Schramm FR. A bioética de proteção: uma ferramenta para a avaliação das práticas sanitárias? Ciênc Saúde Coletiva [Internet]. 2017 [acesso 27 nov 2019];22(5):1531-8. DOI: 10.1590/1413-81232017225.04532017

57. Sinner R. Public theology in Brazil: a first overview. Tor J Theol [Internet]. 2014 [acesso 27 nov 2019];30(1):33-46. DOI: 10.3138/tjt.2534

58. Lange F. Public theology and health care. In: Kim S, Day K, editores. A companion to public theology [Internet] Leiden: Brill; 2017 [acesso 6 jul 2019]. p. 325-46. Disponível: https://bit.ly/30iMXis

59. Schramm FR. Op. cit. p. 1531. 
60. Eich M, Verdi MIM, Finkler M, Martins PPS. Princípios e valores implicados na prática da sedação paliativa e a eutanásia. Interface Comun Saúde Educ [Internet]. 2018 [acesso 27 nov 2019];22(66):733-44. DOI: 10.1590/1807-57622016.0853

61. Eich M, Verdi MIM, Finkler M, Martins PPS. Op. cit. p. 738.

62. Saunders C. Introduction: history and challenge. In: Sykes N, Edmonds P, Wiles J, editores. Management of advanced disease. 4a ed. Londres: Arnold; 2004. p. 3-8.

63. Instituto Brasileiro de Geografia e Estatística. Sinopse do Censo Demográfico 2010 [Internet]. Rio de Janeiro: IBGE; [s.d.] [acesso 25 jun 2019]. Disponível: https://bit.ly/3h7n381

\section{Participação dos autores}

Mary Esperandio redigiu a primeira versão do artigo. Carlo Leget contribuiu com a escrita de algumas seções específicas e com a revisão final. Ambos os autores interagiram ativamente durante a elaboração do estudo e redação do artigo.

\section{Correspondência}

Mary Esperandio - Rua Brigadeiro Franco, 4.185, apt. 11, Rebouças CEP 80220-100. Curitiba/PR, Brasil.

Mary Esperandio - Doutora - mary.esperandio@pucpr.br

(iD) 0000-0001-8521-8794

Carlo Leget - Doutor - c.leget@uvh.nl

(iD) 0000-0002-6647-8141

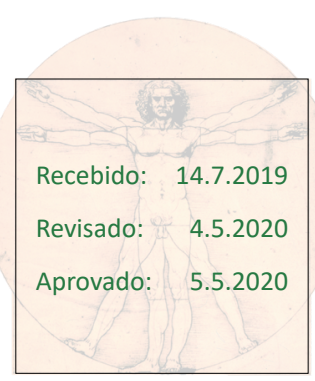

